\title{
Salammbô : Un roman à la limite du roman
}

\author{
Isabelle Daunais
}

Dès sa publication, Salammbô est apparu, à ses principaux commentateurs, comme un roman qui n'en était pas tout à fait un. Pour Sainte-Beuve, Salammbô est un " poème en prose " aussi bien qu'un roman. Théophile Gautier ne voit, quant à lui, aucun mélange des genres : "Ce n'est pas un livre d'histoire, ce n'est pas un roman : c'est un poème en prose » (Le Moniteur officiel, 22 décembre 1862), tandis que George Sand compare le roman à L'Enfer de Dante et parle de Flaubert comme d'un « conteur» (La Presse, 27 janvier 1863) ${ }^{1}$. Cette dernière interprétation n'est pas étonnante tant l'intrigue de Salammbô, psychologiquement peu développée, construite par saillies et relayée par une série d'images fortes perçues comme mémorables au sein même du récit, ressemble à celle des contes. Sa fonction est d'ailleurs plus proche de celle que Walter Benjamin, dans son article sur la figure du « conteur", assignait au récit (ou à la narration) que de celle qu'il attribuait au roman : "D'un côté "le sens de la vie", de l'autre "la morale de l'histoire" ; ces deux mots d'ordre opposent le roman et le récit, et signalent le statut historique entièrement différent que revêt chacune de ces formes d'art ${ }^{2}$ » (dans sa propre traduction de ce texte, Benjamin parle du « sens d'une vie » et de «la morale

${ }^{1}$ Voir Daniel Mortier, « La Réception du roman », Daniel Fauvel et Yvan Leclerc (dir.), Salammbô de Flaubert. Histoire, fiction (Paris : Champion, 1999) 152.

${ }^{2}$ Walter Benjamin, "Le Conteur», trad. de Maurice de Gandillac, Euvres (Paris : Gallimard, coll. «Folio/essais », 2000) 3 :137. 
d'une histoire $\left.{ }^{3} »\right)$. Il est en effet difficile, à propos des personnages de Salammbô, de parler du sens de la vie (et encore moins du sens d'une vie), comme on peut en parler à propos d'Emma Bovary, de Frédéric Moreau ou même de Bouvard et Pécuchet. Il est plus aisé de voir à la fin de Salammbô, une fois le livre refermé, la morale d'une histoire, que ce soit celle de l'orgueil déchu, du triomphe des forts, des lois implacables de la guerre ou, si l'on adopte un point de vue « carthaginois », de la puissance des dieux. On peut aussi, à l'instar de Théophile Gautier et de Sainte-Beuve, conclure que Salammbô, avec ses effets de rythme et de répétition, tient davantage (ou au moins tout autant) du poème en prose que du roman, et que sa matière, aussi concrète soit-elle dans ses éléments particuliers, reste globalement étrangère à la réalité qu'elle cherche à représenter. Bref, il n'est pas facile de reconnaître spontanément Salammbô comme un roman, ou entièrement comme un roman, et plus encore de le reconnaître comme un repère ou un jalon pour notre compréhension de l'art romanesque.

Dans sa réponse aux nombreux reproches que lui adresse SainteBeuve dans son long article du Constitutionnel (8, 15, 22 décembre 1862), Flaubert reconnaît d'ailleurs lui-même, bien que sans le formuler comme tel, la difficulté de lire son roman comme un roman, difficulté qui tient à ce que son héroïne n'est pas ce qu'on reconnaît habituellement comme un personnage romanesque. Au critique qui déplore que Salammbô soit trop romantique, Flaubert répond qu'elle l'est au contraire trop peu :

Elle ressemble selon vous à " une Elvire sentimentale », à Velléda, à Bovary. Mais non! Velléda est active, intelligente, européenne. Mme Bovary est agitée par des passions multiples : Salammbô au contraire demeure clouée par l'idée fixe. C'est une maniaque, une espèce de sainte Thérèse ? N'importe! Je ne suis pas sûr de sa réalité ; car ni moi, ni vous, ni personne, aucun Ancien et aucun Moderne, ne peut connaître la femme orientale par la raison qu'il est impossible de la fréquenter ${ }^{4}$.

Flaubert répète ici une explication qu'il avait déjà formulée dans une lettre à Ernest Feydeau, en août 1859, au moment de la rédaction du roman : «Je commence à voir un peu mes personnages. Je crois qu'ils

${ }^{3}$ Walter Benjamin, « Le Narrateur», Écrits français (Paris : Gallimard, coll. « Bibliothèque des idées ", 1991) 221.

${ }^{4}$ Lettre à Sainte-Beuve, 23-24 décembre 1862, Correspondance, Jean Bruneau (éd.) (Paris : Gallimard, coll. «Bibliothèque de la Pléiade », 1991) 3 : 276-77. La lettre répond aux articles de Sainte-Beuve publiés dans Le Constitutionnel les 8, 15 et 22 décembre 1862. 
ne sont plus maintenant à l'état de mannequins, décorés d'un nom quelconque. Pour qu'on dise d'un personnage antique : "C'est vrai", il faut qu'il soit doué d'une triple vie, car le modèle, le type, qui l'a vu $?^{5}$ » La difficulté de donner vie à des personnages antiques, c'està-dire par définition insaisissables, pour ne pas dire fantomatiques, s'est imposée dès le début à Flaubert comme le grand défi qu'il aurait à relever. Dès le mois de juillet 1857, c'est-à-dire au tout début de l'entreprise, il écrit au même Feydeau : "Je donnerais la demi-rame de notes que j'ai écrites depuis cinq mois et les 98 volumes que j'ai lus, pour être, pendant trois secondes seulement, réellement émotionné par la passion de mes héros ${ }^{6} »$. Or s'il pense, deux ans plus tard, avoir levé la difficulté, sa réponse à Sainte-Beuve montre qu'il a plutôt été contraint de transformer le problème en un trait formel assumé : Salammbô est bel et bien définie par une idée fixe, et il ne faut pas chercher chez elle de psychologie comme on en trouve chez les héros du roman moderne.

Ce que Flaubert se garde cependant de dire à Sainte-Beuve, c'est que la nature de "mannequins » ou de quasi-mannequins de ses personnages pose à ses propres yeux un problème. À deux reprises dans sa correspondance, il recourt au terme de "mannequins " pour parler de personnages qui à ses yeux n'en sont pas. Il l'utilise en 1852 à propos de Graziella de Lamartine, dont les héros, pour être trop évanescents, trop entourés de mystère, trop elliptiques, bref, trop abstraits, ne lui paraissent " pas des êtres humains, mais des mannequins ${ }^{7}$ ». Il utilise également le terme en juillet 1862 à propos des personnages des Misérables de Victor Hugo, qui lui semblent « des mannequins, des bonshommes en sucre ", que "pas une fois on ne [ ... ] voit souffrir, dans le fond de leur âme ${ }^{8}$ ", et il emploie une expression similaire en 1874 à propos de Quatrevingt-treize : «j'aime beaucoup la moitié du premier volume, la marche dans le bois, le débarquement du marquis, et le massacre de la Saint-Barthélemy, ainsi que tous les paysages ; mais quels bonshommes en pain d'épice que ses bonshommes! Tous parlent comme des acteurs. Le don de faire

\footnotetext{
${ }^{5}$ Lettre à Ernest Feydeau, 30 août 1859, Correspondance $3: 38$.

${ }^{6}$ Lettre à Ernest Feydeau, 26? juillet 1857, Correspondance (Paris : Gallimard, coll. "Bibliothèque de la Pléiade », 1980) 2 : 749. Souligné par l'auteur.

${ }^{7}$ Lettre à Louise Colet, 24 avril 1852, Correspondance 2 : 77: «Et d'abord, pour parler clair, la baise-t-il, ou ne la baise-t-il pas? Ce ne sont pas des êtres humains mais des mannequins. - Que c'est beau ces histoires d'amour, où la chose principale est tellement entourée de mystère que l'on ne sait pas à quoi s'en tenir ! "

${ }^{8}$ Lettre à Mme Roger des Genettes, juillet 1862, Correspondance 3 : 236.
} 
des êtres humains manque à ce génie. S'il avait eu ce don-là, Hugo aurait dépassé Shakespeare ${ }^{9}$.»

Certes, ni Mâtho ni Salammbô ne parlent « comme des acteurs » (ne serait-ce que parce qu'ils parlent très peu) et l'on ne saurait dire qu'ils échouent à être des êtres humains, définis et mus qu'ils sont par les grandes forces de l'ambition, de l'orgueil, de l'amour, de la terreur. Mais on chercherait en vain chez eux la qualité romanesque de se transformer au fil du temps, de découvrir en bout de parcours les erreurs et les illusions qui ont été les leurs, de varier dans leur être, de s'étonner de ce qu'ils ont été. Non seulement les données qui les définissent restent stables tout au long du récit, mais nous restons nous-mêmes, comme lecteurs, dans une distance toujours semblable par rapport à eux, distance que crée et maintient l'effet constant d'étonnement qui entoure leurs apparitions, leurs mouvements, et jusqu'à leur présence parmi les autres personnages. Dès lors, peut-on se demander, si ses héros sont peu « romanesques » ou menacent en eux-mêmes la nature romanesque du livre, de quelle façon Salammbô est-il malgré tout, pour Flaubert, un roman, c'est-à-dire parvient, en dépit de cette difficulté centrale, à être un roman ?

La question est semblable à celle que Jacques Neefs et Claude Mouchard posent, dans leur étude de 1986, à propos de Bouvard et Pécuchet. Relevant qu'à force d'érudition, de lectures et de notes, Flaubert s'était « astreint à trop bien savoir » ce qu'il allait trouver au bout de son œuvre, à ne rien y découvrir, les deux critiques s'interrogent, dès lors, sur son choix d'écrire un roman : "Aux imprécations, à l'engueulade généralisée, le Sottisier ou le Dictionnaire des idées reçues (avec sa préface) auraient dû suffire. À quoi bon un roman ? ", question à laquelle ils répondent par cette phrase toute flaubertienne : "C'est un roman, pourtant ${ }^{10}$ ». Ce mot de «pourtant » suggère que quelque chose résiste à ce qui peut être dit ou pensé sous une autre forme, à ce qui ferait du livre autre chose qu'un roman et c'est d'ailleurs en termes de « résistance » que Jacques Neefs et Claude Mouchard définissent la grande trame motrice de l'œuvre, écrivant que tout « ce qui se voit », dans Bouvard et Pécuchet, " tout ce qui arrive, ne se découpe qu'en silhouettes menacées » et qu' « on sent que chaque trait de ce roman a été gagné contre un afflux de puissance indéterminante - et grâce à lui ${ }^{11}$. » Ce terme de "pourtant » et plus largement cette idée de

${ }^{9}$ Lettre à Mme Roger des Genettes, $1^{\text {er }}$ mai 1874, Correspondance (Paris : Gallimard, coll. " Bibliothèque de la Pléiade », 1998) 4 : 793.

${ }^{10}$ Jacques Neefs et Claude Mouchard, Flaubert (Paris : Balland, 1986) 332.

${ }^{11}$ Neefs et Mouchard, Flaubert 332. 
résistance me semblent s'appliquer tout aussi bien à Salammbô, non pour suggérer que le livre puisse ne pas être un roman, mais pour tâcher de saisir ce qui en fait un roman en dépit de l'" idée fixe " de ses personnages, de leur absence de "passions multiples » et de leur réalité problématique.

Comment, donc, Salammbô est-il pourtant un roman ? Pour tenter de répondre à cette question, je propose de recourir à l'expression de «silhouettes menacées » utilisée par Jacques Neefs et Claude Mouchard, qui me semble offrir ici une manière de comprendre comment ce roman, en même temps qu'il déploie une matière qui, à bien des égards, paraît être celle des contes ou de la poésie, travaille presque à chaque page à fragiliser cette matière ou, si l'on préfère, à la ramener du côté du roman.

On peut partir ici du reproche adressé à Flaubert par Sainte-Beuve d'avoir peint dans Salammbô un portrait trop « fabuleux » de Carthage, reproche auquel le romancier avait répondu en se défendant de toute exagération et de tout artifice, arguant qu'une telle perception ne pouvait être que le produit d'un regard occidental :

Pourquoi trouvez-vous que j'ai fait son enfance [à Hannibal] fabuleuse? Est-ce parce qu'il tue un aigle ? Beau miracle dans un pays où les aigles abondent! Si la scène eût été placée dans les Gaules, j'aurais mis un hibou, un loup ou un renard. Mais, Français que vous êtes, vous êtes habitué, malgré vous, à considérer l'aigle comme un oiseau noble, et plutôt comme un symbole que comme un être animé. Ils existent, cependant ${ }^{12}$.

Les aigles existent en effet, mais à la défense de Sainte-Beuve, force est d'admettre que tout concourt, dans Salammbô, à les rendre saisissants, comme sont rendus saisissants l'ensemble des événements, des décors et des objets. Le soin que Flaubert met à les décrire contribue évidemment pour beaucoup à leur relief, mais celui-ci tient plus encore à ce que les personnages eux-mêmes les découvrent dans l'étonnement. Les scènes de saisissement, d'incompréhension, d'effroi, de surprise sont partout présentes dans le roman, où elles reviennent à la manière d'un leitmotiv. Elles procèdent de la nature violente de l'action, qui ébranle même les soldats les plus aguerris, mais aussi de ce que la plupart des mouvements (entrées et sorties des personnages, déplacements) surviennent sans avertissement, créant un effet d'hallucination, l'une des images les plus typiques du roman étant celle où un personnage apparaît devant un autre de manière fantomatique ou terrifiante.

${ }^{12}$ Lettre à Sainte-Beuve, 23-24 décembre 1862, Correspondance 3 : 279. 
On pense à Mâtho qui s'enfuit avec le zaïmph à travers les rues de Carthage : "À l'angle d'un mur, un homme se recula, effrayé par cette chose étincelante, qui traversait les ténèbres "; à Salammbô qui apparaît dans la tente du chef libyen comme elle le ferait sur une scène de théâtre : "Elle arracha les voiles de sa tête. Il se recula, les coudes en arrière, béant, presque terrifié "; à la découverte par les mercenaires des bûchers où les Carthaginois, pour obtenir la faveur des dieux, sacrifient leurs enfants : «Ce grand bruit et cette grande lumière avaient attiré les Barbares au pied des murs ; se cramponnant pour mieux voir sur les débris de l'hélépole, ils regardaient béants d'horreur »; au mercenaire Zarxas qui, après avoir bu le sang de la blessure faite à un ennemi, " entonn[e] une chanson des Baléares, une vague mélodie pleine de modulations prolongées, s'interrompant, alternant, comme des échos qui se répondent dans les montagnes [ . . . ]. Cette chose atroce fit horreur aux Barbares, aux Grecs surtout "; ou encore aux partisans de Hannon et d'Hamilcar venus secrètement avec leurs armes au temple de Moloch et les produisant tout à coup : «Ainsi, par précaution, ils avaient apporté des armes ; c'était un crime ; ils se regardèrent les uns les autres, effrayés. ${ }^{13}$ »

Ces scènes de saisissement ne surviennent pas seulement dans les moments de cruauté ou de sacrilège ; l'étonnement est pour ainsi dire constant dans le roman, où tout apparaît à chacun avec une intensité maximale. Celle-ci s'explique certes par l'état de tension et de fébrilité qui traverse l'ensemble du récit, comme lorsque Hamilcar reprend possession de son palais après la longue absence hors de Carthage : «Le Suffète s'avança dans les appartements déserts. À chaque pas il retrouvait des armures, des meubles, des objets connus qui l'étonnaient cependant ${ }^{14} »$. Mais le saisissement s'explique plus encore par l'identité même de ceux qui découvrent l'action, presque toujours des spectateurs anonymes ou distants, mercenaires ou Carthaginois, à qui tout paraît étonnant : " Des Nomades qui n'avaient jamais vu de ville étaient effrayés par l'ombre des murailles ", "Des Anciens s'étaient postés sur la plate-forme des tours : et l'on ne savait pas pourquoi se tenait ainsi, de place en place, un personnage à barbe longue, dans une attitude rêveuse. De loin, il semblait vague comme un fantôme, et immobile comme des pierres. ${ }^{15}$ »

\footnotetext{
${ }^{13}$ Gustave Flaubert, Salammbô, Gisèle Séginger (éd.) (Paris : Garnier-Flammarion, 2001) 141, 274, 332, 239, 185.

${ }^{14}$ Flaubert, Salammbô 171.

${ }^{15}$ Flaubert, Salammbô 171, 293, 80.
} 
On retrouve bien sûr dans cette manière de faire apparaître l'action la méthode habituelle de Flaubert, certaines formulations circulent même d'un roman à l'autre, produisant des images sinon semblables du moins curieusement apparentées. Par exemple, Catherine Leroux, dans Madame Bovary, apparaît elle aussi, sur l'estrade aux Comices agricoles, comme une figure étonnante que l'on voit se " tenir ainsi », immobile, dans la distance : «C'était la première fois qu'elle se voyait au milieu d'une compagnie si nombreuse ; et, intérieurement, effarouchée par les drapeaux, par les tambours, par les messieurs en habits noirs et par la croix d'honneur du Conseiller, elle demeurait tout immobile, ne sachant s'il fallait s'avancer ou s'enfuir [ ... ]. Ainsi se tenait, devant ces bourgeois épanouis, ce demi-siècle de servitude ${ }^{16}$. " De même, l'effroi des Carthaginois qui se reculent devant la silhouette inattendue que forme Mâtho courant dans la ville en déployant le zaïmph rappelle le saisissement des Rouennais qui, " au coin des bornes ", voient passer " avec de grands yeux ébahis » cette " chose extraordinaire " qu' offre à leur vue le fiacre emportant Emma et Léon dans une course sans but ${ }^{17}$. On pense aussi à Mâtho au moment de son supplice final, alors que la foule le pousse au bas de l'escalier de l'Acropole et que tous les bras se tendent pour le bousculer et le blesser : «L'escalier de l'Acropole avait soixante marches. Il les descendit comme s'il eût roulé dans un torrent, du haut d'une montagne ; trois fois on l'aperçut qui bondissait, puis en bas, il retomba sur ses talons ${ }^{18} »-$ image qui annonce, par la figure étrange qu'elle dessine, celle de Bouvard et Pécuchet s'exerçant au saut à la perche dans la campagne de Chavignolles : " La campagne étant plate, on les apercevait au loin ; - et les villageois se demandaient quelles étaient ces deux choses extraordinaires, bondissant à l'horizon. ${ }^{19}$ "

Ce qui, toutefois, distingue Salammbô des autres romans de Flaubert, c'est la multiplicité et la continuité des regards étonnés par lesquels l'histoire nous est donnée à voir. Ce n'est pas de temps en temps qu'une image surprenante surgit dans le champ de vision d'un personnage ou de spectateurs anonymes, mais presque à chaque instant et comme si c'était la manière principale de raconter. Alors que dans les autres romans l'étonnement est ponctuel (ou, lorsqu'il est

\footnotetext{
${ }^{16}$ Gustave Flaubert, Madame Bovary, Jacques Neefs (éd.) (Paris : Le Livre de poche, 1999) 250-51.

${ }^{17}$ Flaubert, Madame Bovary 372.

${ }^{18}$ Flaubert, Salammbô 374.

${ }^{19}$ Gustave Flaubert, Bouvard et Pécuchet, Claudine Gothot-Mersch (éd.) (Paris : Gallimard, coll. « Folio », 1991) 274.
} 
continu, le fait d'un seul point de vue, comme dans La Tentation de saint Antoine), dans Salammbô il est relayé et pour cette raison comme démultiplié. Venus d'Europe, d'Afrique et d'Asie, les mercenaires découvrent chacun à leur tour des mœurs qu'ils ne connaissent pas, pendant que les Carthaginois s'effraient de leur barbarie généralisée. Or si ce flux tendu de l'étonnement et de l'étrangeté contribue à déporter le roman du côté de la fable - ou encore de la légende ou du songe - il est aussi ce qui en constitue, in fine, sa matière la plus proprement « romanesque ».

Pour saisir cette matière on peut partir de l'une des phrases les plus célèbres du livre, sur laquelle Proust et Albert Thibaudet ont attiré notre attention, autant pour sa beauté grammaticale que pour le sens issu de son rythme, et qui apparaît au début du chapitre XII alors que les mercenaires vaincus enterrent leurs camarades morts au combat, chacun se désolant de ne pouvoir honorer les défunts selon les rites de leur patrie : "Les Latins se désolaient de ne pas recueillir leurs cendres dans les urnes; les Nomades regrettaient la chaleur des sables où les corps se momifient, et les Celtes trois pierres brutes - sous un ciel pluvieux, au fond d'un golfe plein d'îlots. ${ }^{20}$ » Proust admirait dans cette phrase l'usage si typiquement flaubertien du « et » qui, contrairement à l'usage courant, ne fixe pas les choses mais les fait se poursuivre : "C'est comme l'indication qu'une autre partie du tableau commence, que la vague refluante, de nouveau, va se reformer. [ . . . En En mot, chez Flaubert, "et" commence toujours une phrase secondaire et ne termine presque jamais une énumération ${ }^{21}$. " Pour Thibaudet aussi, tout se joue dans le sentiment de prolongement créé par le dernier segment, qui semble se détacher des deux précédents pour mener sa vie propre : "La phrase est jetée dans le moule ternaire coutumier, mais les trois divisions du dernier membre rendent sensibles les trois pierres brutes, les choses mélancoliques et discontinues prolongées encore par cette toile de fond des îlots sur la mer ${ }^{22}$. »

Ce qu'il faut toutefois ajouter aux analyses de Proust et de Thibaudet, pour bien saisir toute la charge représentative de cette phrase, c'est que la mélancolie y est précisément un effet de la secondarité (ou de la discontinuité). Alors que dans Madame Bovary, L'Éducation sentimentale, Bouvard et Pécuchet et plus encore La Tentation de saint Antoine ce sont les personnages principaux qui éprouvent - et qui

\footnotetext{
${ }^{20}$ Flaubert, Salammbô 279.

${ }^{21}$ Marcel Proust, " À propos du style de Flaubert » (NRF, $1^{\text {er }}$ janvier 1920), Essais et articles (Paris : Gallimard, coll. "Folio », 1994) 287.

${ }^{22}$ Albert Thibaudet, Gustave Flaubert [1935, 1922] (Paris: Gallimard, coll. « Tel », 1982) 235 .
} 
éprouvent comme un sentiment décisif - la mélancolie (que ce soit sous la forme de l'ennui, des espoirs déçus, des regrets, du sentiment d'échec), dans Salammbô, la mélancolie, aussi fréquemment évoquée soit-elle, reste secondaire. Et elle l'est non parce que les " héros ", Mâtho et Salammbô, l'éprouvent comme un élément second ou comme un arrière-plan non de leur conscience, mais parce que son foyer est extérieur à eux, qu'il provient de tous ceux dont l'histoire, contrairement à la leur, reste fragmentaire, parcellaire, discontinue. La grande particularité de Salammbô par rapport au reste de l'œuvre flaubertienne tient à ce renversement : ce ne sont pas les personnages principaux qui s'ennuient, regrettent un monde perdu, s'interrogent sur l'avenir ou s'y projettent, bref, pensent à d'autres vies possibles Mâtho et Salammbô sont beaucoup trop captés par leur passion, ou par leur "idée fixe ", pour se concevoir ailleurs que dans le présent de l'action et de leurs vies -, mais les personnages secondaires que sont les soldats et les Carthaginois. Ce sont eux et non pas ceux dont la « passion » nous est racontée qui conservent la mémoire de ce qui n'est plus et, à travers elle, donnent la mesure du temps qui érode les choses :

[L]es palmiers se courbaient, le ciel disparaissait, on entendait rebondir des pierres sur la croupe des animaux ; et le Gaulois [Autharite], les lèvres collées contre les trous de sa tente, râlait d'épuisement et de mélancolie. Il songeait à la senteur des pâturages par les matins d'automne, à des flocons de neige, aux beuglements des aurochs perdus dans le brouillard ; et, fermant les paupières, il croyait apercevoir les feux des longues cabanes, couvertes de paille, trembler sur les marais, au fond des bois.

Tous regrettaient leurs familles, leurs maisons ; les pauvres, leurs cabanes en forme de ruche, avec des coquilles au seuil des portes, un filet suspendu, et les patriciens, leurs grandes salles emplies de ténèbres bleuâtres, quand, à l'heure la plus molle du jour, ils se reposaient, écoutant le vague bruit des rues mêlé au frémissement des feuilles qui s'agitaient dans leurs jardins ; - et, pour mieux descendre dans cette pensée, afin d'en jouir davantage, ils entre-fermaient les paupières ; la secousse d'une blessure les réveillait.

Tout ce qu'il [Hamilcar] avait fait, tout ce qu'il avait vu se déroula dans sa mémoire : les assauts, les incendies, les légions, les tempêtes, Drepanum, Syracuse, Lilybée, le mont Etna, le plateau d'Éryx, cinq ans de batailles, jusqu'au jour funeste où, déposant les armes, on avait perdu la Sicile. Puis il revoyait des bois de citronniers, des pasteurs avec des chèvres sur des montagnes grises ; et son cœur bondissait à l'imagination d'une autre Carthage, établie là-bas ${ }^{23}$. 
Dans l'étude qu'il consacre à Salammbô, Thibaudet s'interroge à son tour sur la nature des personnages qui peuplent le roman. Reprenant la réflexion de Flaubert sur l'impossibilité de représenter des personnages orientaux et tenus par une idée fixe, il écrit, en accord avec lui : "Salammbô est une Orientale, Mâtho est un possédé, et ni l'un ni l'autre ne sauraient être traités selon les procédés d'une psychologie compliquée ». Mais, ajoute-t-il, «Spendius, seul peut-être dans le roman, vit d'une manière complète et que nous reconnaissons, parce qu'ici nous nous trouvons de plain-pied avec le Grec, avec une valeur constante de la vie méditerranéenne et occidentale ». Thibaudet précise qu'un autre élément nous permet aussi de nous attacher au roman et de nous y reconnaître : " Ce qui vit encore avec vraisemblance, intensité et profondeur, ce qui donne à Salammbô cette valeur d'humanité durable qu'il faut bien toujours trouver dans quelque coin d'une belle œuvre, ce sont les ensembles, c'est l'armée des mercenaires et c'est Carthage ${ }^{24}$. " C'est sur ces ensembles que «s'ajuste[nt] l'histoire de Salammbô et l'élément romanesque ${ }^{25}$ ".

On peut formuler les choses un peu différemment : c'est de ces ensembles que naît l'élément romanesque. Ce qui fait que Salammbô n'est pas seulement un "poème en prose ", une fable ou un conte, mais aussi un roman, ce qui fait que l'étonnement permanent qui y a cours ne se fige pas en fantasmagories diverses, ce n'est ni son intrigue ni ses héros, mais l'arrière-plan de ses personnages secondaires. Ce sont eux qui mesurent les transformations et les variations du monde, eux qui en éprouvent la réalité et la perte. Contrairement au couple que forment Mâtho et Salammbô, pour qui tout s'ordonne en fonction de la puissance des dieux et du combat à mort que se livrent Moloch et Tanit, les mercenaires, tout en croyant en leurs dieux, éprouvent aussi le monde comme une chose concrète, soumise à l'infortune, aux erreurs, aux illusions, à la défaite. C'est par eux et à travers leur regard que le monde se présente dans la fragilité et l'incertitude, comme une "silhouette menacée ", pour reprendre à nouveau la belle expression de Jacques Neefs et de Claude Mouchard. C'est par eux et leur humanité très simple que Flaubert construit son roman comme roman, par ces personnages secondaires et discontinus qu'il gagne contre la fixité du conte et de la fable et contre la menace des mannequins qui risquent d'emporter son œuvre. La grande beauté de

${ }^{24}$ Thibaudet, Gustave Flaubert 141.

${ }^{25}$ Thibaudet, Gustave Flaubert 142. 
Salammbô tient à cette résistance, à cette infiltration ou cette conquête du romanesque dans ce qui au départ ne l'est pas - par éloignement, par étrangeté, par impossibilité de savoir. Avec Salammbô, c'est aussi le chemin du roman qui nous est indiqué.

Université McGill 